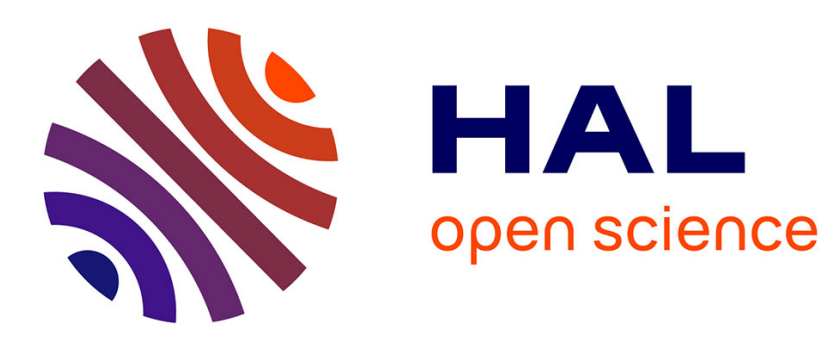

\title{
Désindustrialisation et industrialisation en Algérie. Le rocher de Sisyphe
}

Fatiha Talahite

\section{To cite this version:}

Fatiha Talahite. Désindustrialisation et industrialisation en Algérie. Le rocher de Sisyphe. Outreterre. Revue européenne de géopolitique, 2016, N4, 10.3917/oute1.047.0130 . hal-03323643

\section{HAL Id: hal-03323643 \\ https://hal.science/hal-03323643}

Submitted on 22 Aug 2021

HAL is a multi-disciplinary open access archive for the deposit and dissemination of scientific research documents, whether they are published or not. The documents may come from teaching and research institutions in France or abroad, or from public or private research centers.
L'archive ouverte pluridisciplinaire HAL, est destinée au dépôt et à la diffusion de documents scientifiques de niveau recherche, publiés ou non, émanant des établissements d'enseignement et de recherche français ou étrangers, des laboratoires publics ou privés. 


\title{
DÉSINDUSTRIALISATION ET INDUSTRIALISATION EN ALGÉRIE. LE ROCHER DE SISYPHE
}

Fatiha Talahite

\author{
L'Esprit du temps | «Outre-Terre»
}

2016/2 N 47 | pages 130 à 151

ISSN 1636-3671

ISBN 9782847953701

Article disponible en ligne à l'adresse :

http://www.cairn.info/revue-outre-terre-2016-2-page-130.htm

\section{Pour citer cet article :}

Fatiha Talahite, «Désindustrialisation et industrialisation en Algérie. Le rocher de Sisyphe », Outre-Terre 2016/2 (N47), p. 130-151.

DOI 10.3917/oute1.047.0130

Distribution électronique Cairn.info pour L'Esprit du temps.

(C) L'Esprit du temps. Tous droits réservés pour tous pays.

La reproduction ou représentation de cet article, notamment par photocopie, n'est autorisée que dans les limites des conditions générales d'utilisation du site ou, le cas échéant, des conditions générales de la licence souscrite par votre établissement. Toute autre reproduction ou représentation, en tout ou partie, sous quelque forme et de quelque manière que ce soit, est interdite sauf accord préalable et écrit de l'éditeur, en dehors des cas prévus par la législation en vigueur en France. Il est précisé que son stockage dans une base de données est également interdit. 


\section{Désindustrialisation et industrialisation en Algérie. Le rocher de Sisyphe}

Fatiha Talahite $^{1}$

\section{INTRODUCTION}

Peut-on parler de désindustrialisation en Algérie, sachant qu'il n'y a pas eu dans ce pays de processus abouti d'industrialisation comparable à ce que l'on a pu observer dans les économies émergentes ni même dans certains pays en développement ayant réussi à mettre en place une industrie manufacturière ? Aussi sera-t-il question ici d' " épisodes " industriels, dont le plus consistant fut sans conteste celui qui se déroule entre le milieu des années 1960 et la fin des années 1980. Cet article formule une série d'interrogations sur ces différents moments et avance quelques hypothèses explicatives. L'angle d'approche historique choisi vise à mettre en évidence ce qui rapproche ou distingue ces événements, à dégager les grandes tendances, à identifier les constantes, mais aussi les facteurs de rupture. Se dessine alors un fil conducteur qui suggère dans ces différents épisodes les phases d'un même processus d'industrialisation entrecoupé d'interruptions conduisant à de longues plages de désindustrialisation.

\section{LA DÉSINDUSTRIALISATION EN ALGÉRIE DANS LES ANNÉES 1985-2014}

Lorsqu'on parle de désindustrialisation en Algérie, on se réfère généralement à la période qui a suivi l'épisode le plus long d'industrialisation qu'a connu ce pays, entre le milieu des années 1960 et la fin des années 1980. Dans une étude sur 86 pays pour la période 1975-93, Hossein Jalilian et John Weiss ${ }^{2}$ testent la présence d'un processus de désindustrialisation dans chaque pays, identifiable par l'existence d'un résidu négatif entre la participation effective de l'industrie au PIB et celle anticipée au vu des caractéristiques du pays. Sans surprise ils établissent, d'une part, que l'Afrique a expérimenté durant cette période un degré de désindustrialisation inconnu ailleurs,

\footnotetext{
Économiste, chercheur au CNRS, Cresppa-GTM, UMR 7217 ; <fatiha.talahite@cnrs.fr>

Cf. Hossein Jalilian, John Weiss, "De-industrialization in sub-Saharan Africa : myth or crisis ? ", Journal of African Economies, vol. 9, n 1, 2000, p. 24-43.
} 
d'autre part que l'Algérie fait effectivement partie des pays touchés par une importante désindustrialisation. Si l'on prend la définition la plus courante, on constate en effet que la part de l'industrie dans l'emploi total y a atteint un pic de $18 \%$ en 1977 pour diminuer régulièrement ensuite au profit des services, lesquels s'élèvent à plus de $60 \%$ en 2014. Mais c'est la part de l'emploi dans l'agriculture qui connaît le déclin le plus frappant, de $40 \%$ en 1973 à $9,5 \%$ en 2014 (cf. tableau n ${ }^{\circ} 1$ infra). Pour ce qui est de la part de l'industrie dans la richesse créée, les gains de productivité sont plus rapides dans les services que dans l'industrie (cf. tableau n ${ }^{\circ} 2$ infra). Au niveau symptomatique du moins, on observe bien les principales caractéristiques d'un phénomène de désindustrialisation.

Tableau $n^{\circ} 1$ : Allocation sectorielle de l'emploi dans l'économie (1973-2014)

\begin{tabular}{|l|c|c|c|c|c|}
\hline & 1973 & 1977 & 1987 & 2000 & 2014 \\
\hline Agriculture & $40.0 \%$ & $31,0 \%$ & $18.6 \%$ & $14,1 \%$ & $9,5 \%$ \\
\hline Industrie & $11.2 \%$ & $18,0 \%$ & $16.7 \%$ & $13,4 \%$ & $12,6 \%$ \\
\hline BTP & $8.7 \%$ & $15,5 \%$ & $16.9 \%$ & $10,0 \%$ & $16,5 \%$ \\
\hline Service & $40.1 \%$ & $35,6 \%$ & $47.8 \%$ & $62,5 \%$ & $61,4 \%$ \\
\hline
\end{tabular}

Source : Rafik Bouklia-Hassane in Études sur les perspectives de diversification de l'économie nationale, Forum des chefs d'entreprise, Alger, décembre 2015 (non publié)

Tableau $n^{\circ} 2$ : Transformations structurelles et productivité de l'économie $(1973-87 ; 1987-2014)$

\begin{tabular}{|l|c|c|c|c|}
\hline Secteurs & $\begin{array}{c}\text { Évolution } \\
\text { de la part } \\
\text { d'emploi } \\
1973-87 \\
(\%)\end{array}$ & $\begin{array}{c}\text { Productivité } \\
\text { du travail en } \\
1987 \text { (écart à } \\
\text { la moyenne } \\
\text { nationale*) }\end{array}$ & $\begin{array}{c}\text { Évolution } \\
\text { de la part } \\
\text { d'emploi } \\
1987-2014 \\
(\%)\end{array}$ & $\begin{array}{c}\text { Productivité } \\
\text { du travail en } \\
2014 \text { (écart à } \\
\text { la moyenne } \\
\text { nationale* }\end{array}$ \\
\hline Agriculture & -21.4 & 72 & -9.1 & 1,65 \\
\hline Industrie & +5.5 & 1,08 & -4.1 & 0,59 \\
\hline BTP & +6.8 & 1,06 & 0,4 & 0,93 \\
\hline $\begin{array}{l}\text { Services, } \\
\text { Commerce, } \\
\text { Administration }\end{array}$ & +7.7 & 1,06 & 13.6 & 1,00 \\
\hline
\end{tabular}

* par exemple dans l'agriculture, l'écart de 0,72 signifie que la productivité du travail dans ce secteur au cours de l'année 1987 s'est élevée à $72 \%$ du niveau moyen de productivité observé sur l'économie dans son ensemble

Source : Rafik Bouklia-Hassane in Études sur les perspectives de l'économie nationale, op. cit. 


\section{DÉSINDUSTRIALISATION/INDUSTRIALISATION COLONIALE}

C'est au moment précisément où s'amorçait le développement industriel de la France ${ }^{3}$, à la fin des guerres révolutionnaires et napoléoniennes, que débuta la conquête de l'Algérie en 1830. Quels rapports entre ce processus et la désindustrialisation de la colonie ?4 Contrairement à l'Amérique latine ou à d'autres pays colonisés d'Asie ou d'Afrique (comme l'Afrique du Sud) où l'industrialisation participa d'un enracinement de la minorité européenne ayant débouché sur son autonomisation par rapport à la métropole, les " pieds-noirs " d'Algérie ne développèrent pas de base industrielle propre, ce qui peut expliquer leur départ massif à l'indépendance. En trois brefs moments, sous l'impulsion de la métropole, allait surgir le projet d'industrialiser l'Algérie, chaque fois abandonné pour des raisons essentiellement politiques. Mais auparavant, cette histoire commença par une désindustrialisation brutale et radicale.

\section{Conquête et désindustrialisation PRIMORdiale}

Y a-t-il eu désindustrialisation en Algérie au tout début de la colonisation ? Bien que la réponse factuelle à cette question dépasse le cadre de cet article, elle mérite d'être posée. La recherche en histoire économique montre en effet qu'il existait des formes de proto-industrie dans plusieurs régions de l'Empire ottoman avant la révolution industrielle. Avant la conquête française, l'Algérie faisait partir de ce vaste ensemble et y était rattachée par de nombreux liens économiques. Elle était aussi reliée à l'Afrique subsaharienne par des routes commerciales. La colonisation rompit les liens de l'Algérie avec le vaste espace ottoman et avec l'Afrique dont elle faisait partie et au sein desquels marchands et voyageurs circulaient librement. Même si l'économie côtière était en partie dominée par la Course ${ }^{5}$, il existait des pôles de protoindustrie florissante, notamment dans les villes où s'étaient réfugiées plusieurs vagues de musulmans chassés d'Espagne par les rois catholiques à partir de la fin du $\mathrm{XV}^{\mathrm{e}}$ siècle. On peut ainsi faire l'hypothèse d'une " désindustrialisation " au début du XIXe siècle, conséquence à la fois des destructions liées à la conquête ${ }^{6}$, puis de la concurrence des produits importés de métropole. Parmi les épisodes de la disparition de cette protoindustrie, on peut citer le départ massif d'artisans et commerçants, comme dans le cas de l'« exode de Tlemcen $»^{7}$ vers le Maroc et le Moyen-Orient

\footnotetext{
3 Cf. Maurice Lévy-Leboyer (éd.), Histoire de la France industrielle, Paris, Larousse, 1996.

Cf. Ahmed Henni, La colonisation agraire et le sous-développement en Algérie, Alger, SNED, 1982.

Cf. Merouche Lemnouar, Recherches sur I'Algérie à l'époque ottomane, tome 2: "La course, mythes et réalité ", Paris, Bouchène, 2007, 353 pages.

6 Cf. André-Paul Weber, 1830-1930-La France en Algérie: une malheureuse aventure, Saint-Denis, Éditions Publibook, 2010.

7 Cf. Charles-Robert Ageron, "Les migrations des musulmans algériens et l'exode de Tlemcen (1830-1911) ", Annales Économies, Sociétés, Civilisations, année 1967, vol. 22, n 5, p. 1047-1066.
} 
pour échapper à la conscription obligatoire. Mais cette destruction va plus loin. Le projet colonial, en effet, et plus particulièrement en matière d'industrialisation, fut dès les débuts pensé comme une reconfiguration, un formatage complet de l'économie de la colonie. Tout ce qui avait pu exister avant était frappé de négativité, on ne pouvait rien construire autrement qu'à partir d'une table rase. Il n'y avait rien dans les savoirs et savoir-faire antérieurs qui pût être transmis. Cette attitude caractéristique de l'idéologie industrialiste et moderniste de l'époque est exacerbée dans le cadre colonial. Occultée par l'histoire, que ce soit celle du colonisateur ou de l'Algérie indépendante, cette désindustrialisation première n'en a pas moins marqué l'ensemble du processus et elle est probablement un des éléments d'explication de ses échecs répétés.

\section{INDUSTRIALISATION ET PROJET COLONIAL}

La position des économistes des XVIII et $\mathrm{XIX}^{\mathrm{e}}$ siècles sur la colonisation est paradoxale. Sommairement, si leur doctrine poussait les libéraux à lui préférer le libre commerce entre nations indépendantes, dans lequel résidait à leurs yeux l'intérêt bien compris de tous ${ }^{8}$, les socialistes lui étaient plus ouvertement favorables, au nom de l'idée de " progrès ». Parmi les libéraux, l'école française dite " colonialoéconomique ", avec à sa tête Paul Leroy-Beaulieu (1843-1916), faisait figure d'exception. Elle prônait une colonisation agressive dans le but notamment de fournir à la métropole des matières premières à bas prix et des débouchés pour les produits de son industrie ${ }^{10}$. En principe, les libéraux n'étaient pas fervents de politique industrielle et préféraient laisser agir le marché. Les socialistes, qui visaient à " changer le monde ", se sentaient à l'étroit dans le présent et ils étaient à la recherche d'un espace où réaliser leurs expérimentations ; ils prônaient un engagement plus actif en faveur du « progrès » économique et social. Si les fouriéristes privilégièrent l'agriculture, les saint-simoniens furent les pionniers de l'idée d'industrialisation en Algérie. Alors que pour les libéraux le but premier de l'industrie était de produire des biens en optimalisant l'usage des facteurs de production, les saint-simoniens investissaient celle-ci de vertus modernisatrice et civilisatrices ${ }^{11}$, une vision qui allait perdurer jusqu'après l'indépendance de l'Algérie. Ils défendirent l'idée de développer des infrastructures en les finançant sur fonds publics et trouvèrent un soutien dans la politique du Royaume arabe de Napoléon. Le projet fut abandonné à la chute de

8 Cf. Jean-Philippe Platteau, Les Économistes classiques et le sous-développement, Presses universitaires de Namur, 1978. Ce qui n'empêcha pas certains de s'engager dans la colonisation au nom de la " mission civilisatrice " de l'Occident. À de rares exceptions près, tant les libéraux que les socialistes étaient convaincus de la supériorité de l'Occident et influencés par les théories racistes du XIXe siècle.

9 Cf. Henri Wesseling, The European Colonial Empires, 1815-1919, Harlow, Pearson, 2004.

10 On peut y rattacher également Alexis de Tocqueville et Jules Ferry.

11 Cf. Abdallah Zouache, "Éléments d'économie coloniale saint-simonienne. Le cas de l'Algérie ", Économie et Société, n41, juillet-août 2009 . 
l'empire $^{12}$. En dehors des liaisons télégraphiques (1861), aucun des ambitieux projets de la politique dite de " mise en valeur » - voie ferrée transsaharienne (1879), mer intérieure au Sahara - dans laquelle s'étaient engagés tant Leroy-Beaulieu que les saint-simoniens, ne fut réalisé ${ }^{13}$.

\section{VICHY ET L'ÉCONOMIE ADMINISTRÉE}

Un " Programme général d'industrialisation rationnelle de l'Algérie " fut mis en place en 1941 dans le cadre de la stratégie industrielle de l'État. La sous-industrialisation de la colonie était alors expliquée par l'absence de ressources énergétiques suffisantes, le primat donné à l'agriculture, les réticences de la métropole ${ }^{14}$. Dans le contexte d'un interventionnisme induit par la crise des années 1930, Vichy poussa plus loin la tentative d'une économie dirigée : renforcement des ministères économiques, mise en place d'un lourd appareil de direction et d'orientation de l'économie, contrôle des prix, système de rationnement, développement du corporatisme ${ }^{15}$. Ce programme suscita des inquiétudes en métropole ${ }^{16}$. Il fut fait appel aux grands groupes métropolitains ${ }^{17}$ mais la rupture avec la métropole en novembre 1942, en coupant ces entreprises de leurs directions, freina l'élan d'industrialisation amorcé. Cette stratégie ne parvint pas à enrayer le processus de dégradation de l'économie algérienne : baisse de la production agricole, pénuries (textile, charbon), recul des importations. L'excédent de la balance commerciale révélant le poids de la contribution de l'Algérie au ravitaillement de la métropole.

\section{L'industrie dans le Plan de Constantine}

La troisième fois qu'un projet d'industrialisation est engagé en Algérie, c'est avec le Plan de Constantine. En 1955, le rapport Maspétiol évalue les investissements nécessaires au développement de la colonie, en se rapportant à d'autres pays de taille et de développement comparables. Il servira d'hypothèse aux Perspectives décennales du développement économique de l'Algérie élaboré en 1957 par une équipe de jeunes hauts fonctionnaires et de dirigeants d'entreprises, puis au Plan de Constan-

\footnotetext{
12 Lorsque le républicain Jules Ferry déclare que "La politique coloniale est fille de la politique industrielle ", c'est l'industrialisation de la métropole qu'il a d'abord en vue.

13 Cf. Henri Wesseling, The European Colonial Empires, 1815-1919, op.cit.

14 Cf. Jacques Cantier, L’Algérie sous le régime de Vichy, Paris, Odile Jacob, 2002.

15 Les musulmans sont sous-représentés, seuls quelques grands notables siègent dans les comités.

16 " II ne faut pas que ce pays s'équipe industriellement [et] se cantonne dans une autarcie néfaste pour elle et pour nous ", s'inquiète le président de la Chambre de commerce de Marseille, Jacques Cantier, L'Algérie sous le régime de Vichy, op. cit.

17 Pont à Mousson (usine métallurgique, Oran), Saint-Gobain (verreries d'Afrique du Nord, Oran), ciments Lafarge (Alger), création d'une Société chimique nord-africaine.
} 
tine (1957-61). Dans la filiation des plans quinquennaux français, ce dernier s'inspirait de ceux conçus par Jean Monnet puis Pierre Massé pour la Reconstruction en métropole après 1945. Mais il était surtout marqué, dans sa dimension sociale, par la préoccupation de l'État français de pacifier la colonie. Visant explicitement à contrecarrer l'influence de la résistance au sein de la population algérienne, il prétendait affaiblir les aspirations nationalistes par le développement économique. Il concernait tous les secteurs (industrie lourde, industrie légère, agriculture, défense et restauration des sols, hydraulique, routes et ports, logement, enseignement, action sociale). Dans la sidérurgie, quatre unités à capitaux français produisant surtout pour l'agriculture, avec 150000 tonnes de produits sidérurgiques par an, satisfaisaient les besoins locaux. Elles employaient 1500 ouvriers et tout l'encadrement était européen. Le Plan projetait une unité d'exportation d'acier à Duzerville (futur complexe d'El Hadjar) pour les chantiers navals du Sud de la France (La Ciotat, La Seyne) notamment, d'où sa localisation près d'un port ; cette période est aussi celle de la découverte et du début de l'exploitation du pétrole (1956), ce qui explique l'intérêt nouveau de l'État pour l'économie de la colonie : développement d'infrastructures, construction d'oléoducs, mise en place d'une industrie lourde. Le Plan ne dura que trois ans. Des projets étaient à peine lancés dans l'industrie lourde. Les réalisations furent plus importantes dans le social et, dans une moindre mesure, les infrastructures, que dans l'industrie. Ce fait mérite qu'on s'y arrête. C'est à cette période en effet que se produit en France l'expansion de l'État-providence, dont la définition est étroitement liée à la citoyenneté. Mais qu’en est-il précisément de cette dernière en Algérie, alors département français ? On sait que les musulmans, soumis au code de l'indigénat, en sont exclus en vertu du sénatus-consulte de 1865 qui soumet la jouissance des droits de citoyen au statut personnel. Or dans cette période des changements politiques et juridiques importants s'amorcent. La loi Lamine Guèye de mai 1946, reprise par la Constitution de 1946, reconnaît la citoyenneté française à " tous les ressortissants des territoires d'outre-mer (Algérie comprise) ", dissociant ainsi la citoyenneté de sa dépendance au Code civil pour la rattacher à la nationalité. Le droit de vote est élargi à tous les Algériens de plus de 21 ans. Ces dispositions ne seront jamais appliquées. En septembre 1947, un statut spécifique est voté pour l'Algérie, qui la définit comme " un groupe de départements dotés de la personnalité civile, de l'autonomie financière, et d'une organisation particulière ». Censé introduire l'égalité politique et civique et un égal accès aux fonctions publiques, il maintient inchangé le principe du double collège (une voix d'électeur du premier collège en valait huit du deuxième collège $)^{18}$. Les Algériens ne sont plus des « indigènes ", mais des "Français musulmans ", citoyens de statut local par opposition aux "Français non-musulmans ", citoyens de statut civil. Les différences de droit entre ces deux catégories ne sont plus justifiées par la différence de statuts, mais par une

18 Alors que l'ordonnance du 2 août 1945 accordait aux femmes le droit de vote en France, l'Assemblée algérienne née du statut de 1947 le refuse aux Algériennes. 
distinction entre les deux territoires. Le déclenchement de la guerre d'indépendance le $1^{\text {er }}$ novembre 1954 relance le processus : à partir de 1956, l'État entreprend la dissolution progressive des institutions élues sous le régime du double collège ; les ordonnances du 15 novembre 1958 abolissent le lien entre citoyenneté et Code civil, suppriment les deux collèges électoraux, fusionnent les populations en une seule catégorie de " Français à part entière » et accordent à la population algérienne une représentation politique proportionnelle à son importance dans chaque département algérien. Mais ces transformations, qui exacerbent la colère des pieds-noirs lequels voient se réduire leurs privilèges, viennent trop tard. En 1959, Le président de Gaulle se prononce pour l'autodétermination de l'Algérie. L'indépendance est proclamée en 1962, ouvrant sur une nouvelle nationalité et sur l'espoir d'une pleine citoyenneté, promesse non remplie à ce jour. La mise en relation des événements politiques et économiques de cette période nous permet de faire l'hypothèse que le Plan de Constantine s'inscrivait aussi dans ce processus ; du moins en reflétait-il les contradictions, visant à la fois à désamorcer la radicalisation du mouvement national, à intégrer progressivement les Algériens dans une forme de citoyenneté et un embryon d'État-providence tout en relançant le projet d'équipement et d'industrialisation de la colonie ${ }^{19}$. L'intensification du conflit, la menace pour les pieds-noirs de perdre leurs privilèges et finalement l'option pour l'autodétermination de l'Algérie provoquent une fuite des capitaux. Ce court épisode d'industrialisation sera suivi d'une phase de désindustrialisation provoquée par la guerre puis par le départ massif des Européens au moment de l'indépendance. Les projets en cours seront repris à l'indépendance : sidérurgie à Annaba, liquéfaction du gaz naturel à Arzew (Compagnie Algérienne du Méthane Liquéfié, CAMEL), mise en chantier d'un troisième oléoduc. Cet héritage colonial marquera l'orientation de la politique industrielle de l'Algérie indépendante, notamment par le poids accordé à la sidérurgie.

\section{INDÉPENDANCE, DÉSINDUSTRIALISATION, INDUSTRIALISATION}

En 1962, l'Algérie hérite d'une économie dévastée. Conséquence de la guerre et des sabotages systématiques des groupes politiques ultras qui avaient complètement désorganisé la vie économique, la désindustrialisation est aggravée par le départ massif en France d'industriels, entrepreneurs, cadres et employés, notamment parmi la main-d'œuvre qualifiée, en majorité des Européen ${ }^{20}$. Ce départ a aussi pour effet de réduire considérablement le marché intérieur des produits industriels, adapté sur-

19 En France, la baisse du poids de l'industrie dans la valeur ajoutée apparaît au milieu des années 1960, et la baisse de l'emploi industriel date du milieu des années 1970.

20 Parmi eux des Algériens qui avaient collaboré avec la puissance coloniale (harkis) ou d'autres qui firent le choix de partir face à l'incertitude quant à leur emploi et leur revenu. 
tout aux besoins et aux goûts des Européens. Même dans les industries de grande consommation comme le textile où de nouvelles unités sont en voie d'achèvement, les entreprises existantes ne trouvent pas dans le marché local actuel des débouchés suffisants. Une des premières mesures adoptée par le gouvernement est une hausse générale des salaires. Mais elle ne suffit pas à relancer la consommation et se répercute surtout sur les prix de revient. Tandis que le marché des produits industriels se réduit, les approvisionnements en matières premières deviennent aléatoires. L'Algérie est tributaire de l'étranger, en particulier de la France, et les circuits, qui dépendent largement de la métropole, sont perturbés voire interrompus. Les délais pour obtenir des fournitures s'allongent et les conditions financières se durcissent. Le système financier est totalement désorganisé. En 1962 les dépôts bancaires diminuent d'environ $40 \%$. Le crédit devient cher et difficile à obtenir.

La désindustrialisation se poursuit avant que le pays ne s'engage dans un ambitieux projet de développement. En 1962, le Programme de Tripoli met l'accent sur le rôle prédominant des industries de base (désignées comme "industrie lourde "), notamment les hydrocarbures et la sidérurgie ${ }^{21}$. Cette stratégie ne sera pas mise en place immédiatement. À ce moment, rien n'est encore tranché concernant la politique industrielle à mettre en place, notamment quant au rôle du secteur privé et des investisseurs étrangers. Dans les premières années, le gouvernement adopte un plan d'équipement annuel. Incomplètement réalisé, il ne permet pas une relance efficace de l'économie et le taux d'activité industrielle continue à baisser (de 10\% à $75 \%$ selon les secteurs). L'héritage du Plan de Constantine, le mouvement d'autogestion dans l'agriculture et l'industrie, mais surtout la rupture avec la métropole qui s'apparente côté français à un embargo larvé joueront un rôle décisif sur l'orientation qui sera prise ensuite.

\section{L'AUTOGESTION}

D’abord une réponse spontanée au problème des « biens vacants » agricoles et industriels, l'autogestion, décrétée en mars 1963, ne s'appliquait qu'aux biens abandonnés par leurs propriétaires européens ${ }^{22}$. Durant l'année 1963, les entreprises vacantes ou dont l'activité n'était pas jugée suffisante sont confiées aux travailleurs dirigés par un comité de gestion élu par eux. En octobre 1963, le gouvernement décide la liquidation totale de la propriété foncière coloniale et un certain nombre

21 "Le développement réel et à long terme du pays est lié à l'implantation des industries de base nécessaires aux besoins d'une agriculture moderne. À cet égard, I'Algérie offre de grandes possibilités pour les industries pétrolières et sidérurgiques. Dans ce domaine, il appartient à l'État de réunir les conditions nécessaires à la création d'une industrie lourde ", ministère de l'Information, Programme de Tripoli, Dossiers documentaires n²4, Alger, janvier 1976, p. 49.

22 Cf. Hélie Damien, "L'autogestion industrielle en Algérie ", Revue de l'Occident musulman et de la Méditerranée, $1969, n^{\circ} 6$, p. 113-126. 
de nationalisations dans l'industrie. Les entreprises étatisées deviennent sociétés nationales et sont regroupées avec les entreprises autogérées en un seul "secteur socialiste " et à partir de fin 1963 il n'y aura plus de mises en autogestion. Le secteur autogéré comprend en majorité des petites entreprises et ateliers artisanaux dont l'intérêt économique n'est pas toujours évident, souvent maintenus pour des raisons sociales. Toutes les entreprises abandonnées n'ont d'ailleurs pu être remises en activité et près de 300 sur 700 sont restées fermées. Il y a peu de moyennes entreprises (45 dans l'Algérois, 22 dans l'Oranais, 12 dans le Constantinois employant plus de 20 ouvriers) et les grandes entreprises sont quasi inexistantes (moins de 5 emploient plus de 100 ouvriers). L'importance du secteur autogéré est réduite par rapport à l'ensemble de l'industrie. Hors les travaux publics et le bâtiment, il ne représente que $25 \%$ des 60000 ouvriers employés par l'industrie. Le personnel comprend très peu de cadres ou ouvriers qualifiés ${ }^{23}$ par rapport au nombre élevé d'ouvriers non qualifiés. Les entreprises autogérées ne peuvent accorder aux cadres des salaires aussi élevés que dans le privé ${ }^{24}$. Le contrôle de l'administration, qui ne s'est établi que peu à peu, a mis fin aux hausses de salaires décidées par les entreprises qui avaient atteint $30 \%$ dans le secteur autogéré. La baisse du niveau de la production est très importante. Dans les branches les plus atteintes se posent des questions de reconversion d'activité. Le gouvernement préfère maintenir artificiellement en activité des usines qui devraient être mises en faillite, mais ce secteur ne bénéficiera pas d'un apport en cadres ni de l'appui de l'administration. Elles n'ont pas accès au crédit du fait de son coût mais aussi parce que leur nouveau statut juridique n'entre pas dans le cadre de la législation bancaire. Les conditions dans lesquelles s'est déroulée cette expérience expliquent en grande partie qu'elle n'ait pas contribué à mobiliser les travailleurs pour relancer l'industrie. Cet échec a discrédité pour longtemps l'idée autogestionnaire en Algérie.

\section{INDUSTRIALISATION ET MODĖLE DE DÉVELOPPEMENT AUTOCENTRÉ (1966-1986)}

Le modèle algérien de développement s'inspire formellement de l'histoire économique de l'Europe des XVIII ${ }^{\mathrm{e}}$-XIX ${ }^{\mathrm{e}}$ siècles, interprétée comme une succession de révolutions (industrielle, scientifique et technique). Laccent mis sur la rupture avec le passé sonne comme un écho à la table rase de la conquête. On pense que le développement suit une trajectoire unique et que l'on peut en accélérer le rythme d'une façon volontariste. L’industrie est à la base de cette stratégie de développement

23 Les cadres techniques et administratifs mais aussi les contremaîtres, chefs d'équipe et ouvriers qualifiés, en majorité européens, sont partis en même temps que les patrons.

24 Une égalisation des salaires entre les différents secteurs est revendiquée par le Congrès de l'autogestion industrielle de mars 1964. 
accéléré dans laquelle la technologie joue un rôle central. Il en découle la conviction que le développement industriel nécessite des entreprises de grande taille ainsi que l'introduction de technologies modernes. Un rôle central est accordé à l'État comme agent de rupture et de modernisation, plus important même que celui qu'il a pu jouer historiquement en Europe car justifié par la faiblesse ou l'absence d'une classe sociale porteuse de la modernité2 ${ }^{25}$. Ces questions ont souvent été en débat - à la fois sur le rôle de l'industrie, de la technologie, de l'État et des différentes forces sociales dans le développement - et cela a parfois donné lieu à plusieurs expérimentations voire à des inflexions dans la stratégie de développement. La référence à la théorie de la dépendance justifie le choix d'un modèle autocentré. Elle combine une représentation de l'économie mondiale en termes de centre-périphérie à une théorie du sous-développement causé par la domination impérialiste, laquelle se perpétue par l'échange inégal qui permet le prélèvement d'un surplus croissant au profit du centre. L'industrialisation, nécessaire pour rompre cette dépendance, s'inscrit dans une stratégie de rupture avec le marché mondial capitaliste, dans le cadre d'une économie nationale protégée ${ }^{26}$. Cette rupture, qui correspondait certes au contexte politique et intellectuel de l'époque, s'est surtout imposée à l'Algérie du fait de la manière dont s'est opérée la décolonisation. Dans une telle perspective, l'autarcie était conçue pour être durable. Présentée officiellement pour la première fois dans le Rapport de présentation du budget d'équipement 1966, la stratégie industrielle à long terme, sur 15 ans (1966-1980), visait à "l'autonomie technologique ", conçue comme le fait de concevoir ses propres équipements et de transformer ses produits industriels. La priorité est donnée aux produits de base, avant tout la sidérurgie et la pétrochimie. L'objectif d'un mode d'accumulation autonome est construit en référence au modèle soviétique d'autarcie mais aussi à celui de petits pays ${ }^{27}$ d'Europe de l'Ouest (Autriche, Espagne). Notons qu'en prenant ces économies comme modèles, le décideur négligeait le facteur taille, pourtant essentiel : l'URSS (tout comme la Chine d'ailleurs), pouvaient soutenir l'option d'une certaine autarcie, d'autant que cette économie appartenait à un ensemble plus vaste, le Comecon; de même que les petits pays d'Europe de l'Ouest s'intégraient à l'espace de la CEE. Aussi, pour suivre l'un ou l'autre de ces modèles, il aurait fallu projeter la stratégie de développement sur une échelle plus large que le cadre étroit du marché national et songer dès cette époque à la constitution d'un espace économique régional intégré (cadre maghrébin, arabe, africain ${ }^{28}$. Inspiré de la théorie des industries industrialisantes de Gérard Destanne de Bernis ${ }^{29}$. Le modèle se démarque à la fois de la théorie de la croissance

25 Cf. Djamel Guerid, L'exception algérienne : la modernisation à l'épreuve de la société, Alger, Casbah édition, 2007.

26 Cette conception se distingue de celle de la théorie des industries naissantes au XIX siècle (List) qui ne préconisait la protection que comme instrument provisoire dans la phase de naissance et de maturation des industries nouvelles.

27 Comme l'avait fait le rapport Maspétiol, mais pas dans une perspective d'autarcie, au contraire il s'agissait alors d'arrimer le développement industriel de l'Algérie à l'économie de la métropole.

28 Cf. Malek Bennabi, L'Afro-Asiatisme, 1956, avait formulé ce projet à l'occasion de la Conférence de Bandung.

29 Elle-même issue d'une tradition qu'on peut faire remonter à la théorie du " développement déséquilibré " de Hirschman, qui se transmet par François Perroux et intègre des principes du modèle soviétique. 
équilibrée de Ragnar Nurkse mais également du modèle d'import-substitution qui met la priorité sur les biens de consommation. Dans la définition qu'elle donne des "industries de base ", socle de l'industrialisation, la Charte nationale (1976) retient les mêmes branches que de Bernis : métallurgie, fabrications mécaniques, électriques et électroniques, constructions navales, pétrochimie et chimie des produits de base, des industries qui « jouent un rôle stratégique déterminant puisqu’elles assurent l'indépendance de l'industrie nationale, et partant, l'indépendance du pays tout court ". Cependant, leur rôle dans le modèle diffère de celui des industries industrialisantes (ou du modèle soviétique) notamment parce qu'il n'est pas accordé de priorité absolue à une branche par rapport à une autre. Toute industrie considérée comme favorable à l'indépendance nationale pourra être intégrée.

Dans les faits la priorité sera accordée à deux branches, la sidérurgie et la pétrochimie. Selon un schéma élaboré à partir d'une certaine lecture de la "Révolution industrielle » européenne, on attend de la première une forte impulsion en aval sur la production agricole, les industries métalliques et mécaniques, le BTP (inputs à base d'acier). La sidérurgie, qui, dans le modèle soviétique comme dans celui de Destanne de Bernis, est le noyau dur des industries qui procurent le développement, a acquis un caractère mythique, ce qui peut expliquer certaines décisions irréalistes la concernant. Ainsi, le complexe sidérurgique d'El Hadjar (charpentes, ronds à béton) est devenu le symbole de l'épopée industrielle algérienne. Une autre croyance issue de la "Révolution industrielle " était que l'industrialisation passait nécessairement par l'implantation d'entreprises de grande taille. Cette option ne se limite pas à l'industrie lourde, elle est élargie aux industries de base, à l'industrie textile et la mécanique de précision, avec la construction de très grands complexes hautement capitalistiques. Ce choix du planificateur en faveur d'unités fortement intégrées, s'il peut s'expliquer initialement par l'absence de tissu industriel, sera ensuite accentué par le caractère étatique et centralisé des investissements ainsi que la concentration par l'État de très larges moyens de financement. Le modèle se caractérise enfin par sa structure en étapes : d'abord assurer les conditions de base (sidérurgie, pétrochimie) par la mise en place de grandes capacités de production (acier, engrais, énergie, ciment) ; puis moderniser le secteur traditionnel, notamment agricole, grâce à la production par ces industries de base d'inputs pour le secteur agricole (tracteurs, camions, machines agricoles, machines-outils). Les deux objectifs - construire une économie indépendante par l'édification d'industries de base et assurer les besoins de la population par des industries de transformation - s'ils ne sont pas hiérarchisés $a$ priori, le sont de fait avec la primauté accordée aux industries de base. Cependant, il y a un chaînon manquant dans ce processus. Dans la théorie de Destanne de Bernis en effet, comme dans le modèle soviétique, les industries de biens d'équipement définis comme ceux qui entrent dans la Formation brute de capital fixe (FBCF) des entreprises - jouent un rôle central dans la reproduction de l'ensemble du système 
productif. Depuis les schémas de la reproduction de Marx, ce secteur est au cœur des stratégies d'industrialisation visant l'introversion. Or on ne les retrouve pas dans le modèle algérien ${ }^{30}$. Comptabilisées dans l'industrie de base ou l'industrie d'intégration, leur rôle n'est pas distingué de celui des matériaux de construction ou de l'acier. Dans le plan, ces biens relèvent de la branche des industries sidérurgiques, métalliques, et électriques (ISME), regroupant la mécanique lourde et légère, l'électricité et l'électronique. En fait, en dehors d'investissements pour l'agriculture et le BTP, il n'y a pas de programmation de production de biens d'équipement pour l'industrie, ce qui signifie que l'on compte durablement sur les importations. On peut supposer que, compte tenu du caractère « étapiste » du modèle, l'investissement dans ce secteur a été implicitement reporté à une programmation ultérieure. Mais ce chaînon manquant dans la stratégie industrielle algérienne fait planer un doute sur la volonté proclamée d'ériger un système industriel totalement autonome. Ainsi, malgré la témérité des objectifs annoncés, il semble qu’à l'origine le planificateur ait posé des limites au modèle, intériorisant d'une certaine manière une fatalité du sous-développement lui interdisant de pousser jusqu'au bout l'ambition du développement industriel sous sa forme autocentrée. On aurait pu y voir un certain réalisme quant à l'impossibilité d'un développement totalement autocentré, mais il aurait alors fallu penser les liens d'interdépendance avec les pays de la sous-région par exemple dans le cadre d'un développement mutuel. Cette faille a ouvert la brèche à une dépendance croissante des importations du Nord.

\section{Place centrale des hydrocarbures et déclin de l'agriculture}

La réalisation de cette stratégie s'est faite en trois grandes phases :

1) 1967-73. Mise en place du modèle autocentré : nationalisations, constitution des grands ensembles industriels, élaboration des plans de développement ${ }^{31}$, l'année 1971 étant marquée par des événements (nationalisation des hydrocarbures, lancement de la Révolution agraire et de la Gestion socialiste des entreprises) qui vont accentuer l'orientation socialiste.

2) 1974-79. La hausse du prix du pétrole et l'augmentation des capacités financières du pays outre des besoins planifiés provoquent une forte accélération de l'investissement industriel, induisant un recours croissant à l'assistance technique étrangère et à l'endettement extérieur qui lui est lié, au-delà des capacités d'absorption de l'économie, avec des "restes à réaliser ", de plus en plus importants à la fin

30 Cf. Mahrez Hadjseyd, L'Industrie algérienne. Crise et tentative d'ajustement, Paris, I'Harmattan, 1996.

31 Succession des plans de développement : plan triennal ou pré-plan (1967-69) ; premier plan quadriennal (1970-73) ; deuxième plan quadriennal (1974-77) ; puis " pause " dans les Investissements, investissements " hors plan " et " Reste à Réaliser » (1978-79) ; premier plan quinquennal (1980-84) ; deuxième plan quinquennal (1985-89), abandonné peu après son lancement à cause de la crise de 1985 . 
de chaque plan. Cette période se caractérise par l'ampleur de l'investissement. Entre la première et la dernière année du premier plan quadriennal (1970-1973), il est multiplié par 1,5, puis par 2,2 de 1973 à 1977. Mesuré en pourcentage du PIB, le taux d'investissement est de $28,3 \%$ durant le premier quadriennal, de $40,4 \%$ pour le second et il atteint des pics de $42,6 \%$ en 1977 et de $47,8 \%$ en 1978, année au cours de laquelle il a été le plus élevé dans le monde. La part des infrastructures économiques augmente (elle passe de 1,14 à 11 milliards de dinars algériens (DA) entre 1967-1969 et 1980-1984) tandis que celle de l'agriculture stagne (1,8 milliards de DA constants 1984 par an).

3) À partir de 1979, la croissance projetée était trop importante pour les capacités d'absorption existantes. Le taux d'investissement connaît un net fléchissement en 1978-1979, suggérant une pause pour parachever les projets en retard et réaliser ceux qui n'ont pu l'être. Par ailleurs, si, au cours de la période 1967-1979, l'investissement public était dominé par l'investissement industriel, notamment dans les hydrocarbures, celui-ci sera, à partir de 1980, principalement alloué aux infrastructures économiques et sociales (ouvrages d'art, barrages hydrauliques, routes, chemins de fer, santé, éducation, administration). Par ailleurs, le taux d'investissement (FBCF/PIB) pour l'ensemble des secteurs est un record absolu, tant par rapport aux pays industrialisés qu'à ceux en développement ${ }^{32}$. Le dispositif de planification en place, et plus précisément le mécanisme d'ajustement ex ante à un niveau centralisé, n'ont pas permis d'opérer une réorientation de ces moyens financiers vers un développement soutenu.

La prédiction de De Bernis d'une forte impulsion de la sidérurgie et de l'industrie de biens d'équipement sur la production agricole n'a pas eu lieu. L'agriculture entrera dans un déclin dont l'un des facteurs explicatifs est la disponibilité des revenus pétroliers. Mais d'un autre côté, on voit mal comment l'Algérie aurait pu ne pas utiliser ses ressources en hydrocarbures pour son développement. On peut poser la question autrement et s'interroger sur l'absence d'un surplus agricole pour financer le développement ${ }^{33}$. L'agriculture algérienne avait été déstructurée par la politique coloniale. Spécialisée dans la production de vin (il représente jusqu'au quart de la production française pendant la période coloniale), elle voit se fermer son principal débouché, le marché français ${ }^{34}$. En termes de main-d'œuvre, l'exode rural causé par l'expropriation et la prolétarisation de la paysannerie algérienne s'est intensifié pendant la guerre. À la suite du départ massif des colons, l'opportunité d'une

32 Cf. Christian Palloix, "Industrialisation et financement lors des deux plans quinquennaux (1970-1977) ", Tiers-Monde, 1980 , tome $21, n^{\circ} 83,1980$, p. 531-555.

33 Cet état de fait consacra définitivement la suprématie des "industrialistes " sur les " agrariens " dans le débat des débuts de l'indépendance sur le choix d'une stratégie de développement.

34 Pendant la colonisation, plusieurs crises de surproduction dans I'Hexagone avaient provoqué I'hostilité des viticulteurs français aux vins d'Algérie, contre lesquels fut créée l'AOC ils étaient exclus. 
expérience autogestionnaire ${ }^{35}$ dans les exploitations agricoles modernes des grandes plaines du Nord s'est soldée par un échec. Si le projet algérien de développement n'accordait pas un rôle moteur à l'agriculture, la modernisation de ce secteur est pourtant un objectif stratégique à moyen terme, visant tout à la fois à assurer l'indépendance alimentaire, à stimuler les exportations agricoles et à élever le niveau de vie dans les campagnes. Dans le modèle autocentré, cela passe par l'apport en produits industriels tels que machines et engrais, ce qui justifie la priorité accordée à l'industrie. Le rôle secondaire de l'agriculture dans le processus de développement s'est traduit par une faiblesse des investissements par rapport à l'industrie. Si officiellement les besoins en équipement de l'agriculture sont couverts à $80 \%$ par les capacités installées de l'industrie mécanique, cela ne concerne que le secteur public. En réalité, le taux de mécanisation baisse en raison de l'insuffisance de la production des complexes de machinisme agricole et de la pénurie de pièces de rechange. Un exemple d'intégration industrie-agriculture est la production d'engrais. L'idée est de moderniser l'agriculture tout en valorisant le phosphate et l'éthane (produit du gaz naturel), jusque-là exportés à l'état brut. C'est l'un des premiers investissements programmés et réalisés après l'indépendance. L'usine d'Arzew, construite en 1968, était déjà prévue par le Plan de Constantine. Des investissements importants sont faits dans de grands complexes d'engrais (1,5 milliards de dollars US pour 5000 emplois créés) mais ceux-ci sont peu performants du fait de problèmes de gestion liés à leur taille ainsi que de choix technologiques erronés (fermeture de l'unité d'Arzew en 1977). Tandis que des excédents difficiles à exporter s'accumulent, la production de l'engrais le plus demandé (le triple super phosphate, TSP) est insuffisante et on a recours à l'importation. Dans l'autre sens, la relation agriculture-industrie se limite à la production agricole pour l'industrie, notamment agroalimentaire (conserveries, huileries, sucreries, semouleries, minoteries, etc.). Dans cette branche où le secteur privé, relativement bien implanté, est plus efficace que le public, les difficultés d'approvisionnement, la sous-utilisation des capacités et l'insuffisance de la production par rapport aux besoins croissants ${ }^{36}$ amènent à recourir de plus en plus aux importations d'inputs (produits agricoles) et de produits finis.

On entre ainsi dans un cercle vicieux où le recours aux importations pour satisfaire la demande sociale permet de pallier les incohérences et les limites du modèle, ce qui accentue l'extraversion du système (abandon de certaines cultures et productions concurrencées par les importations). La facture alimentaire (20\% des importations) finira par absorber la totalité des disponibilités monétaires mobilisées après paiement de la dette. Les ressources pétrolières ont permis d'avoir recours aux importations pour faire face à une demande de produits agricoles en hausse, du fait

35 Cf. Claudine Chaulet, La Mitidja autogérée: enquête sur les exploitations autogérées agricoles d'une région d'Algérie, 1968-1970, Alger, SNED, 1971.

36 La part de l'agriculture dans le PIB chute de $22 \%$ à $8 \%$ entre les plans triennal et quadriennal. 
de la croissance démographique. D’autant qu'en raison du dumping pratiqué par les pays de l'Organisation de coopération et de développement économiques (OCDE, subventions aux exportations ou à la production), ces biens étaient disponibles sur le marché mondial à un prix souvent inférieur à ce qu'il coûtait sur place. Cette dépendance alimentaire n'a cessé depuis de s'approfondir, faisant de l'Algérie l'un des plus gros importateurs de blé au monde. Le déclin de l'agriculture s'est accompagné d'un large mouvement d'exode rural, déjà amorcé pendant la colonisation ${ }^{37}$.

\section{Bref biLAN de L'industrialisation}

Tableau $n^{\circ} 3$ : Principaux indicateurs du développement industriel (\%)

\begin{tabular}{|l|c|c|c|}
\hline Indicateurs de développement industriel & $1963-86$ & $1987-1999$ & $2000-2005$ \\
\hline VA industrielle/le PIB & 12,6 & 10,8 & 6,6 \\
\hline $\begin{array}{l}\text { VA industrielle publique/VA } \\
\text { industrielle totale }\end{array}$ & 74,1 & 77,6 & 65,1 \\
\hline $\begin{array}{l}\text { Variation de la production industrielle } \\
\text { publique en volume }\end{array}$ & 11,2 & $-2,7$ & 0,0 \\
\hline Emploi industriel /emploi total & 12,2 & 10,3 & 7,4 \\
\hline $\begin{array}{l}\text { Importation produits industriels/ } \\
\text { importations totales }\end{array}$ & 90,2 & 86,9 & 89,0 \\
\hline $\begin{array}{l}\text { Exportation produits industriels/ } \\
\text { exportations totales }\end{array}$ & 7,8 & 4,3 & 3,0 \\
\hline $\begin{array}{l}\text { Variation moyenne des prix à la } \\
\text { production industrielle publique }\end{array}$ & - & 23,8 & 3,5 \\
\hline $\begin{array}{l}\text { Variation moyenne des prix à la } \\
\text { production industrielle privée }\end{array}$ & - & 15,7 & 0,8 \\
\hline
\end{tabular}

Source : Comptes économiques, Office national des statistiques (ONS), différentes années

Le tableau n 3 supra reprend les indicateurs liés à l'investissement sur l'ensemble de la période et illustre le poids de l'industrie tout autant que la montée en puissance puis le déclin en fin de période de l'investissement public industriel. Sur une période de plus de 15 ans, la croissance industrielle est à deux chiffres et la part de la valeur ajoutée de l'industrie manufacturière dans le PIB (hors hydrocarbures, énergie et mines) atteint $16 \%$ en 1970-1973. Trois branches réalisent plus de $70 \%$ de toute la

37 Cf. Abdellatif Benachenhou, L'exode rural en Algérie, Alger, SNED, 1979. 
valeur ajoutée industrielle : l'agroalimentaire (27\%), les ISME (22\%) et le textilehabillement $(22 \%)$. L'essentiel de la production industrielle est assuré par les entreprises publiques. Leur part dans la production industrielle globale passe de $80 \%$ à $85 \%$ entre 1969 et 1978, bien que leurs performances restent en deçà des capacités de production installées, utilisées à $50 \%$ environ. Durant la phase intensive de mise en place de l'appareil de production industrielle (1967-1979) il n'y a pas de gains de productivité. Au contraire, celle-ci chute de 15 points environ par rapport à 1969 dans l'industrie hors hydrocarbures. Par contre, la productivité apparente du travail (valeur ajoutée par emploi en DA constants 1984) passe de l'indice 85 à 137 entre 1979 et 1984 (base 1969) et double pratiquement pour l'ensemble de l'économie. Mais bien que la période 1980-1984 ait été la meilleure pour l'industrie hors hydrocarbures du point de vue de la production et de la valeur ajoutée, la productivité de l'industrie est, dans toutes les périodes, inférieure à celle de l'ensemble de l'économie, quel que soit le niveau des prix des hydrocarbures ${ }^{38}$. Enfin les exportations de produits industriels, qui se limitent à quelques excédents de la sidérurgie, sont insignifiantes (600 millions de DA par an, soit 120 millions de dollars US). Si au vu des objectifs du modèle ce résultat ne peut être considéré comme négatif, car la production industrielle y était destinée non à l'exportation mais au marché intérieur, il l'est, et de façon dramatique, par ses effets sur la balance commerciale, les importations d'intrants pour les besoins de l'appareil de production industriel, y compris ceux d'origine agricole, représentant pour la période $56 \%$ des importations.

Dans le modèle les industries de base, considérées comme le "socle " de l'industrialisation, étaient diversifiées (métallurgie, fabrications mécaniques, électriques et électroniques, constructions navales, pétrochimie et chimie des produits de base) et aucune priorité absolue n'était accordée à une branche par rapport à une autre, mais dans les faits, la branche des hydrocarbures - en particulier son sous-secteur exportateur - fut privilégiée. La pétrochimie (hors gaz naturel liquéfié, GNL) reçut peu d'investissements et les biens d'équipement encore moins. Le projet du Plan de Constantine pour la sidérurgie en tant qu'industrie de base est repris et intégré au modèle orienté vers la demande domestique. Surdimensionné par rapport aux besoins locaux, sa vocation exportatrice est d'abord partiellement maintenue à un moment où le marché mondial de l'acier entre en crise. Puis la situation s'inverse avec l'expansion rapide de la demande locale, dopée par les choix industriels (construction de grandes structures fortement consommatrices d'acier et tendance à la surconsommation). En 1989, moins de la moitié de la demande est couverte par la production locale. Mais ce succès est à relativiser au vu de l'endettement de la branche ainsi que des coûts de production. L'investissement, qui atteint $20 \%$ de l'investissement industriel hors hydrocarbures et $10 \%$ de l'investissement industriel 
total pour 1967-1989, dépasse 3000 dollars US par tonne installée, soit plus du double des investissements similaires dans le monde. Malgré une sous-utilisation des capacités de production, l'Algérie doit importer de l'acier, ce qui révèle la rigidité du modèle orienté vers la satisfaction de la demande locale et montre comment il entre en contradiction avec l'objectif d'indépendance économique. Dans les ISME, l'industrie mécanique occupe une place centrale (électroménager, électronique grand public, produits sanitaires, menuiserie, aluminium). La plupart des unités, réalisées "produit en main ", ont un faible degré de maîtrise technologique et d'autonomie par rapport aux concepteurs étrangers et accusent d'importants déficits d'exploitation, avec une faible utilisation des capacités de production. L'investissement global dans les biens d'équipement représente $6 \%$ de l'investissement industriel global et $12 \%$ de l'investissement industriel hors hydrocarbures (prix constants 1984). La moitié concerne les biens d'équipement mécaniques, ce qui est peu par rapport aux montants alloués à l'industrie. On relève des retards importants dans la réalisation des projets (19,4 mois en moyenne de dépassement des plannings des prévisions), l'insuffisance du taux de couverture de la demande par la production locale, une faible utilisation des capacités de production (en 1982 les industries mécaniques ne réalisent pas plus de $20 \%$ de la production planifiée), une production peu diversifiée. Bien qu'amorçant une baisse continue après 1978, les importations restent élevées (elles atteignent en 1987 le niveau de 1972). Malgré la chute des investissements après 1985 , leur poids relatif dans le total des importations ne diminue pas significativement.

En termes de valorisation des ressources naturelles, l'ambition était de rompre avec la spécialisation de type colonial (exportation de matières premières versus importation de produits industriels) tout en édifiant une industrie utilisatrice de ressources locales abondantes et à bas prix ${ }^{39}$. À plus ou moins long terme, on vise à mettre fin à toute exportation de matière première brute grâce à l'installation d'industries de transformation capables d'absorber la totalité de la production minière. Au niveau de la filière hydrocarbures, le résultat, bien que limité, est tangible : $15 \%$ des hydrocarbures liquides produits sont transformés sur place ; $60 \%$ du pétrole brut sont utilisés par l'industrie pétrochimique ; $20 \%$ de la production de gaz naturel sont utilisés à des fins domestiques par l'industrie et les ménages. Hormis le gaz naturel, les exportations d'hydrocarbures tendent à diminuer et celles de produits raffinés à se stabiliser autour de $20 \%$ de la valeur des exportations d'hydrocarbures. Cependant, si une amorce de remontée de filière est perceptible, l'intégration interbranches est plus problématique, comme pour l'intégration hydrocarbures-agriculture, avec les engrais. Au niveau des exportations, la part des hydrocarbures est passée de $84 \%$ en 1967-1979 à 97 \% en 1980-1989, du fait bien sûr de la hausse des cours sur les mar-

39 La référence est plutôt la théorie smithienne des coûts absolus que l'avantage comparatif ricardien. 
chés mondiaux, mais aussi de l'effondrement de l'exportation des produits agricoles et autres produits miniers (phosphate, minerai de fer).

Entre 1967 et 1989, 2540000 emplois nouveaux ont été créés, dont 460000 industriels. Le taux de chômage passe de $23 \%$ à $14 \%$ au cours de la décennie 1970 . Mais dans les années 1980, malgré une création d'emploi supérieure, il remonte à son niveau des premières années d'indépendance ( $23 \%$ en moyenne). Les sureffectifs importants ( 15 à $30 \%$ des effectifs globaux) s'expliquent par le fait que l'allocation du travail reste dominée par des logiques sociales plutôt qu'économiques. L'emploi pléthorique dans l'administration et le secteur public pèse sur la productivité du travail, tandis que gonfle la part des inactifs dans la population (jeunes en marge du système scolaire, femmes à la maison). D'autant que l'émigration du travail vers la France est officiellement arrêtée depuis 1974. La création de postes de travail a un effet sur la consommation des ménages qui augmente de 4,4\% en 1965-1980. Mais le coût de création d'un emploi dans le secteur public, calculé en rapportant les emplois créés jusqu'en 1989 aux investissements publics réalisés de 1967 à 1989, est très élevé. Les investissements massifs opérés durant cette période participent d'un processus de transformation accélérée de l'économie et de la société où la scolarisation massive, notamment des filles, et le développement du système éducatif, l'urbanisation, l'augmentation des revenus, l'émergence d'une classe moyenne, comme le bouleversement des modes de consommation et des modèles culturels se heurtent rapidement aux limites du marché national. Le secteur privé essentiellement des petites et moyennes entreprises, s'il ne disparaît pas, conservera un rôle marginal, et, privé d'un cadre concurrentiel, il sera condamné à capter des rentes générées par l'État ${ }^{40}$.

Dans la stratégie algérienne de développement, la priorité a été mise sur l'industrie, considérée comme seule à même de propulser rapidement le pays dans la modernité. Les revenus des hydrocarbures ont assuré le financement d'un vaste programme d'investissements dans des industries placées sous la tutelle de l'État et consacrées au marché intérieur. Cette stratégie fut accélérée par l'augmentation du prix du pétrole en 1973 et l'afflux de revenus d'exportation, au point de sortir de ses rails. Si la priorité est donnée à l'industrie lourde (sidérurgie, métallurgie), l'objectif est l'édification d'un système productif national intégré. Cependant, tandis que les entreprises sont largement dépendantes des importations pour leurs équipements et leurs intrants, seul le secteur des hydrocarbures est tourné vers l'exportation. On comprend dès lors les difficultés qu'il y aura à reconvertir ce système pour diversifier les exportations et pourquoi l'ouverture de l'économie aura pour effet de renforcer la part des hydrocarbures dans le PIB. Peut-on dire que l'ajustement par le marché

40 Cf. Brad L. Dillman, State and Private Sector in Algeria : the Politics of Rent-Seeking and Failed Development, San Franscisco (Cal.)-Oxford, Boulder, Westview Press, 2000. 
aurait été mieux adapté pour réagir aux chocs pétroliers externes et plus généralement aux fluctuations des ressources financières extérieures propres à une économie pétrolière ? Il est vrai que, vu leur ampleur, ces chocs ont eu aussi des effets négatifs dans des pays pétroliers où opérait l'ajustement par le marché - effets analysés en termes de Dutch Disease (exploitation de ressources naturelles combinée au déclin de l'industrie manufacturières) - avec cependant une plus grande capacité de réaction et d'adaptation.

\section{Choc pétrolier de 1985-1986, réforme (1988-1991) Et AJUSTEMENT STRUCTUREL (1994-1996)}

Le modèle, en attribuant un rôle central à l'industrie, visait à créer des irréversibilités. Or, confronté à des chocs, il s'est avéré réversible. L'insolvabilité de l'économie, révélée par le choc pétrolier de 1985-1986, apparaît comme une sanction externe de ses faibles performances en termes de compétitivité internationale. Dans un premier temps, et malgré une forte réduction des importations dès 1985, le choc a peu d'effets sur le niveau de la production, en raison de stocks importants accumulés auparavant. Pour se prémunir contre les aléas bureaucratiques et éviter les ruptures cycliques, les entreprises constituaient des stocks d'inputs qui tournaient autour de trois ans en moyenne. Coûteux pour l'entreprise, ils ont contribué à différer les effets de la crise, mais en les aggravant. La croissance ralentit mais la production ne commence à baisser significativement qu'après 1990. Jusqu'en 1987, elle se maintient et même s'améliore dans certaines branches, puis décline progressivement, notamment dans les ISME. Alors que la création d'emploi ralentit dès 1985, avec des compressions d'effectifs dans la Petite et moyenne industrie (PMI) publique et privée, on enregistre peu de pertes d'emploi dans le secteur public industriel national, où des facilités de caisse sont accordées aux entreprises en difficulté afin qu'elles puissent continuer à verser les salaires, en attendant la fin de la crise. En l'absence de traitement social du chômage, la solution était de maintenir une main- d'œuvre pléthorique dans les entreprises et les administrations. La dimension mythique de l'industrialisation interdisait de toucher aux symboles que représentaient les grands complexes industriels. En octobre 1988, El Hadjar, fleuron de l'industrialisation, entre dans une suite de conflits sociaux très durs et interminables tandis que les résultats de l'entreprise se dégradent. Sur deux millions attendus, la production chute à 760000 tonnes. Les salaires n'étant pas calculés en fonction des résultats financiers de l'entreprise mais du volume de la production physique, les entreprises publiques étaient incitées à minorer la baisse de leur production. Leurs résultats sont de moins en moins en rapport avec leur production. Il en résulte une augmentation des stocks de produits finis (qui atteindront un niveau alarmant après 1990) et une détérioration du niveau réel des prix, minés par l'inflation. 
En 1985-1989, la PIB baisse de 2,4 \% par an par rapport à 1980-1984, tandis que la production de l'industrie manufacturière chute de 3,3\%. Mais surtout, la valeur ajoutée industrielle diminue de 6,3 \% par an, entraînant celle des services $(-4,2 \%)$ et du BTP $(-2,8 \%)$, alors qu'elle progresse dans l'agriculture $(+4,8 \%)$, probablement en raison de la redistribution des terres en 1987. Entre 1980-1984 et 1985-1989 l'investissement global moyen (en DA constants) baisse de $52 \%$ et l'investissement industriel de 56,5\%. Cette baisse atteint $68 \%$ dans les hydrocarbures, le secteur le plus touché, qui passent de $16 \%$ de l'investissement public global durant le plan de 1980-1984 à $10 \%$ en 1985-1989 (ils étaient à $29 \%$ en 1967-1979) et tombent pour la première fois en dessous de $45 \%$ de l'investissement industriel (environ $35 \%$ ). La baisse est moindre pour l'industrie hors hydrocarbures ( $46 \%$ ). La chute de 26,1 \% du taux d'investissement provoque un assèchement des recettes en devises des entreprises publiques et une réduction de leurs possibilités de financement extérieur qui les contraint à recourir aux crédits à court terme. Par contre, après 1985, nombre de projets de moyenne importance sont réalisés par le secteur privé (350 unités de production dans les matériaux de construction, l'agroalimentaire, la sous-traitance) ; ils représentent $28,5 \%$ des investissements.

La réforme initiée en 1988-1989 en Algérie ${ }^{41}$ s’inscrit dans la transformation postsocialiste. Elle vise à mettre fin à la tutelle de l'État sur l'économie et à rétablir les institutions du marché, mais aussi à préserver le système industriel en l'aidant à s'adapter et devenir performant. Les mesures adoptées dès janvier 1988 touchent les entreprises publiques économiques (EPE) avec la loi sur l'autonomie de l'entreprise ${ }^{42}$. Contrairement à l'ajustement structurel, qui aura lieu plus tard, en 1994-1996, et conduira au démantèlement partiel du système industriel, elle ne précipite pas les privatisations. La transformation du capital des EPE en " titres de participation " gérés par des "Fonds de participation " publics est une tentative de stimulation du marché et une étape vers leur ouverture progressive à des actionnaires privés, nationaux ou étrangers. Ces mesures se heurtent alors à de fortes résistances. La réforme vise à rétablir la concurrence et plus globalement des mécanismes de marché dans l'économie. Une nouvelle législation supprime le cloisonnement entre secteurs juridiques public et privé. Désormais, toutes les entreprises sont soumises aux mêmes règles et aux mêmes contraintes, notamment dans leurs relations avec les banques. La loi sur les faillites abat le mythe de la pérennité des entreprises publiques. Mais la dynamique sociale et politique à la base de ces changements institutionnels est brisée avec l'interruption de la réforme économique à la mi-1991 et surtout le coup d'État de janvier 1992 qui met fin au processus de démocratisation. Même si les nouvelles règles ne sont pas remises en cause dans leur totalité, elles prendront un caractère de

41 Cf. Fatiha Talahite, " 25 ans de transformation post-socialiste en Algérie ", Tiers-Monde, n 210, 2/2012, août 2012.

42 Cf. Mustapha Menouer "L'autonomie de l'entreprise publique en Algérie. La fin de l'hégémonie étatique ", Annuaire de l'Afrique du Nord, tome XXX, CNRS Éditions, 1991. 
plus en plus formel. En 1994-1999, le rééchelonnement de la dette soumet l'Algérie à un programme d'ajustement structurel. Les privatisations touchent d'abord les entreprises du secteur manufacturier ainsi que le BTP, induisant d'importantes destructions d'emploi ${ }^{43}$. Mais pour les grands ensembles industriels ${ }^{44}$, le processus de privatisation traînera en longueur et sera finalement interrompu avec la remontée des prix du pétrole, dans les années 2000. Dès que la situation financière le permet à nouveau, le gouvernement se remet à injecter des ressources financières dans les entreprises publiques industrielles, renouant avec les coûteux et interminables plans de restructuration qui avaient jalonné la deuxième moitié des années 1980 sans effets positifs notoires. Viennent s'ajouter à cela les programmes de "mise à niveau " avec l'aide de l'UE, dans le cadre du partenariat euro-méditerranéen.

\section{CONCLUSION}

Le processus de désindustrialisation observé en Algérie depuis la fin des années 1980 et qui se poursuit jusqu'à aujourd'hui est complexe et a probablement plusieurs causes. On peut y voir des effets liés à la transition postsocialiste (1988-1991), à l'ajustement structurel (1994-1995), au Dutch Disease et même à la mise en place d'une forme d'État-providence. L'Algérie ayant connu une expansion exceptionnelle de son industrie durant la période socialiste, la désindustrialisation qui a suivi pourrait être attribuée à la transition. La question serait alors de savoir si les politiques de réforme puis d'ajustement structurel ont été " efficaces » ou " inefficaces » en termes d'allocation des ressources, notamment du travail, entre les secteurs ? Pour y répondre, il faudrait pouvoir isoler ces effets, et pour cela disposer de données fiables et précises sur l'évolution de l'emploi et de la productivité par secteur. Cependant, le processus de déclin de l'industrie avait commencé avant la mise en œuvre des réformes. L'effet Dutch Disease est probablement le plus massif ${ }^{45}$, surtout depuis la hausse exceptionnelle du prix du pétrole après 2008, consécutivement à la croissance chinoise. S'il a pu être relativement maitrisé par la politique de taux de change de la Banque d'Algérie, celle-ci n'a pas été à ce jour accompagnée d'une stratégie industrielle suffisamment cohérente et efficace ${ }^{46}$ pour amorcer une relance de l'industrie. À ce stade, l'analyse devrait être complétée par une démarche d'économie politique ${ }^{47}$. Enfin, l'angle d'approche historique a permis de révéler une dépendance de sentier, en montrant comment les ferments de la désindustrialisation étaient inscrits dans

43 Cf. Rafik Bouklia-Hassane, Fatiha Talahite, "Marché du travail, régulation et croissance économique en Algérie ", Tiers-Monde, $\mathrm{n}^{\circ} 194$, avril-juin 2008.

44 Le complexe sidérurgique d'El Hadjar, racheté par I'Indien Mittal, sera récupéré par l'État algérien en 2015.

45 Impossible à identifier pour la période socialiste, car construit sur un modèle d'économie ouverte de marché.

46 Cf. Rafik Bouklia-Hassane, Fatiha Talahite, Les politiques industrielles en Algérie (1963-2007), Madrid, Casa Arabe, 2008.

47 Cf. Rachid Mira, Économie politique de l'industrialisation en en Algérie. Analyse institutionnelle en longue période, thèse de doctorat en sciences économiques de I'Université de Paris 13, décembre 2015. 
le mode d'industrialisation et ses contradictions internes. Issue de la reconversion du Plan de Constantine - qui visait à arrimer l'industrie algérienne aux besoins de la métropole - elle est engagée au moment d'une rupture avec la France s'apparentant à un blocus économique, qui pousse l'Algérie à vouloir relever le défi en se retranchant dans un repli autarcique. Les projets déjà engagés par le Plan de Constantine (1954-1956) sont repris après une interruption de dix ans (1956-1966) et intégrés à un modèle d'industrialisation autocentrée qui se révélera insoutenable. Cet épisode appartient désormais à l'histoire. 